\title{
Direct Measurement of the Capillary Pressure Characteristics of Water-Air-Gas Diffusion Layer Systems for PEM Fuel Cells
}

Jeff T. Gostick, Marios A. Ioannidis*, Michael W. Fowler and Mark D. Pritzker

University of Waterloo, Department of Chemical Engineering, Waterloo, ON N2L 5N5

*Corresponding Author: mioannid@uwaterloo.ca 


\section{Abstract}

A method and apparatus for measuring the relationship between air-water capillary pressure and water saturation in PEMFC gas diffusion layers is described. Capillary pressure data for water injection and withdrawal from typical GDL materials are obtained, which demonstrate permanent hysteresis between water intrusion and water withdrawal. Capillary pressure, defined as the difference between the water and gas pressures at equilibrium, is positive during water injection and negative during water withdrawal. The results contribute to the understanding of liquid water behavior in GDL materials which is necessary for the development of effective PEMFC water management strategies.

Keywords: capillary pressure, hysteresis, water management. 


\section{Introduction}

Further reduction of the mass-transport-induced voltage loss at high current density is critically needed for broad commercial application of polymer electrolyte membrane fuel cells (PEMFC) [1]. Membrane electrode assemblies (MEAs), gas diffusion layers (GDLs) and flow fields must be designed to synergistically provide efficient removal of product liquid water and improved access of reactant gases to catalytic sites. Notwithstanding recent efforts, the capillary properties of GDL-water-air systems have not been conclusively established and their dependence on GDL pore structure and wettability remains unclear.

The capillary pressure of interest in water-air-GDL systems is the difference between the pressures of the liquid and gas phases, $P_{C}=P_{L}-P_{G}$, across the static air-water interfaces within a GDL. It is fundamentally related to the mean curvature $H$ of the air-water interface through the well-known Young-Laplace equation [2]:

$$
P_{C}=\sigma \nabla \cdot \vec{n}=2 \sigma H
$$

where $\vec{n}$ is the unit normal to the surface and $\sigma$ is the surface tension. The shape of the static air-water interface (thus $H$ ) is obtained from the solution of Eq.(1) subject to a boundary condition provided by the equilibrium contact angle $\theta$. The solid surface is said to be hydrophobic for $\theta>90^{\circ}$ (measured through the aqueous phase) and hydrophilic otherwise. Fibrous GDL materials are treated to various extents with poly(tetrafluoroethylene) (PTFE) to render their internal surfaces more hydrophobic. The contact angle of water-air interfaces on internal GDL surfaces has been indirectly estimated to be in the range $88^{\circ}<\theta<101^{\circ}$ for treated and untreated GDLs [3, 4], whereas values of $108^{\circ}$ and $86^{\circ}$ have been reported for 
water on smooth PTFE and water on smooth graphite, respectively [5]. At capillary equilibrium, all air-water interfaces within a GDL have the same mean curvature $H$. Capillary equilibrium of water and air within GDL pores can be established over a broad range of water saturations $S_{W}$ by changing the phase pressure difference $P_{L}-P_{G}$ in a step-wise fashion. The resulting functional relationship $P_{C}\left(S_{W}\right)$ between capillary pressure and water saturation is not unique [6]. Permanent hysteresis is observed because capillary equilibrium is reached via a sequence of non-equilibrium interfacial configurations which differ depending on the history of saturation change [6].

Recent attempts to determine $P_{C}\left(S_{w}\right)$ for water and air in GDLs by different methods have not been entirely satisfactory. A first attempt by Gostick et al. [7] using the method of standard porosimetry (MSP) provided capillary pressure data along a path of decreasing water saturation. Water withdrawal occurred at negative capillary pressures, which was attributed to the existence of hydrophilic pore networks. Others [8] found that a positive capillary pressure is required for water injection. Fairweather et al. [9] reported capillary pressure hysteresis with $P_{C}>0$ for water injection and $P_{C}<0$ for water withdrawal. Contrary to expectation, no hysteresis was observed in the measurements of Nguyen et al. [10].

In this communication, we report a straightforward method for measuring the water-air $P_{C}\left(S_{w}\right)$ relationship for GDL materials along any saturation path in the range $0 \leq S_{w} \leq 1$. Data obtained with this method elucidate the capillary behavior of water-air-GDL systems. 


\section{Experimental Method}

This method controls the gas pressure in the sample to impose constant capillary pressure values and monitors the resulting change in water saturation. The system consists of an analytical balance (Denver P-314), a syringe pump (Harvard 11-Plus), an absolute pressure gauge (Omega PX303-030A5V) and a specially designed sample holder.

\subsection{Sample Holder}

The sample holder shown in Figure 1 was built in-house. The holder features a porous hydrophobic membrane (Sartorius, $0.45 \mu \mathrm{m}$ PTFE \#11806-25) above the sample and a porous hydrophilic membrane (Millipore, $0.22 \mu m$ PVDF \#GVWP04700) below the sample, which act as capillary barriers. The hydrophobic membrane above the sample allows air to escape as water enters the GDL but prevents water from leaving the system after breakthrough of the sample. The hydrophilic membrane below the sample serves the same function at highly negative capillary pressures by allowing water, but not air, to exit. A key feature of this setup is that the sample can be maintained at $P_{C} \approx-10,000 \mathrm{~Pa}$ during assembly which enables the measurement of capillary curves beginning with the GDL in a fully dry state, which serves as a reference for tracking the sample saturation. The holder is designed to hold samples $0.01905 \mathrm{~m}$ in diameter. For a typical material, this sample size corresponds to a pore volume of about $85 \mu \mathrm{L}$, or $85 \mathrm{mg}$ of water, meaning that an analytical balance with resolution of $0.1 \mathrm{mg}$ can detect very slight saturation changes. 


\subsection{Sample Mounting}

The piping and cavity in the base plate are first primed with de-ionized and de-gassed liquid water. This is followed by positioning the liquid distributor made of 20 holes of $800 \mu m$ diameter. The hydrophilic membrane is laid on top of the liquid distributor along with the membrane locating gasket. Next the intermediate plate is bolted to the base plate to hold the hydrophilic membrane tightly in place. At this point, $-10,000 \mathrm{~Pa}$ of suction is applied to system through a hose connected to the liquid port. This suction drains free water from the sample cavity and creates a $-10,000 \mathrm{~Pa}$ capillary pressure at the surface of the hydrophilic membrane. The dry GDL is then loaded with the sample locating gasket. Next, the hydrophobic membrane, compressing cylinder, plug and spring are inserted. The plug prevents bulging of the hydrophobic membrane at high capillary pressures, which would create the appearance of extra water volume in the system. The spring applies only a slight pressure to the plug to prevent GDL compression. Finally, the top plate is bolted to the intermediate plate to apply a downward force on the compression cylinder to seal the assembly.

\subsection{System Setup}

After mounting the sample, a gas pressure of $10,000 \mathrm{~Pa}$ is applied to the gas port. This pressure, combined with the liquid suction at the liquid port, temporarily creates $P_{C}=-20,000$ $\mathrm{Pa}$ in the hydrophilic membrane which is still well below its bubble point. After the gas pressure is established, the liquid suction is relieved and the sample chamber is connected to the water reservoir on the balance. Since the water reservoir is only slightly below the sample, the liquid suction is relatively small and so a positive gas pressure must first be 
applied to maintain the net $P_{C}=-10,000 \mathrm{~Pa}$. This assembly and setup ensures that the GDL never contacts water with a capillary pressure greater than $-10,000 \mathrm{~Pa}$. To verify that the sample does not take up any water during assembly, the above assembly and setup steps were repeated several times followed by immediate disassembly and weighing of the samples.

\subsection{Procedure}

Following system setup, a syringe pump is connected to the gas port of the sample holder. The pump compresses and expands the gas above the sample, thereby effecting changes in capillary pressure. The syringe pump and balance are controlled through an RS-232 interface. After each change in gas volume and therefore capillary pressure, the mass of liquid on the balance is monitored for change. The system is held at a constant capillary pressure until the water mass reading on the balance is stable. The transient responses of the water uptake to changes in capillary pressure (see Figure 2) show that it is indeed stable before each subsequent change in gas pressure.

\subsection{Data Analysis}

Capillary pressure is controlled by adjusting the gas pressure above the sample and is determined as follows. The distance $h$ between the liquid reservoir level and the sample surface is $10 \mathrm{~cm}$, creating a small amount of liquid suction. Also, the barometric pressure $\left(P_{\text {ATM }}\right)$ acts on the surface of the liquid reservoir, but not on the liquid in the sample since the gas chamber is sealed. The capillary pressure is therefore given by: 


$$
P_{C}=P_{L}-P_{G}=\rho g h+P_{A T M}-P_{G}
$$

Controlling capillary pressure by controlling gas instead of liquid pressure is helpful, since most liquid pressure sensors exhibit some membrane displacement that could be incorrectly construed as sample pore volume. Although liquid pressure does vary during periods of transient water uptake due to viscous effects, it returns to its original value, $P_{L}$, once the saturation stabilizes and flow ceases.

Since the sample is initially dry, water uptake by the sample equals water loss from the reservoir. The water saturation is computed as:

$$
S_{W}=\frac{V_{W}}{V_{P}}=\frac{m_{W} \cdot \rho^{-1}}{\frac{\pi}{4} d^{2} \delta \cdot \varepsilon}
$$

where $V_{w}$ is the volume of water in the sample, $V_{P}$ is the GDL pore volume, $m_{w}$ is the mass of water in the sample, $\rho$ is the density of water, $d$ is the sample diameter, $\delta$ is the sample thickness and $\varepsilon$ is the sample porosity. The thickness of each sample was measured directly with a micrometer while porosity was found from mercury intrusion porosimetry tests on samples taken from the same sheet [7]. Evaporative loss of water from the system during the course of a run (ca. $5 \mathrm{hr}$ ) is minimized by covering the top of the beaker, leaving only a small hole for the tubing and also by maintaining the gas above the sample fully humidified. Nevertheless, a small correction to $m_{w}$ is still made by determining the rate of evaporation $(1 \pm 0.2 \mu \mathrm{g} / \mathrm{s})$ during runs with no GDL sample. In experiments with GDL samples, the time to reach capillary equilibrium at each point is recorded and then the corresponding 
evaporative loss is found as the product of equilibration time and evaporation rate. 


\section{Results and Discussion}

Figure 3 shows the capillary pressure curves for water injection and withdrawal, including internal hysteresis loops, for Toray090 (0\% PTFE, $\varepsilon=0.78, \delta=295 \mu \mathrm{m})$ and SGL10BA (5\% PTFE, $\varepsilon=0.88, \delta=360 \mu \mathrm{m})$. No water penetrates the samples before $P_{C}$ reaches positive values of about 1,000 $\mathrm{Pa}$ for Toray090 and about 2,000 $\mathrm{Pa}$ for SGL10BA. A saturation plateau is reached at approximately $10,000 \mathrm{~Pa}$ for both samples which extends to $30,000 \mathrm{~Pa}$. At $P_{C}=30,000 \mathrm{~Pa}$, Toray090 is fully saturated, whereas SGL10BA exhibits a saturation of 0.84. The inability to completely fill SGL10BA can be attributed to microscopic roughness caused by binder and PTFE additives. It might, however, be due to errors in the calculation of $V_{P}$ in Eq.(3) because of uncertainty in $\varepsilon$ or $\delta$ due to inadvertent sample compression during assembly. The fact that Toray090, which is free of rough binder and PTFE, did reach the expected saturation suggests that roughness is the likely explanation. Upon reducing the capillary pressure, $S_{w}$ remains virtually unchanged until negative capillary pressures are reached. Water begins to withdraw from SGL10BA at $P_{C} \approx-1,500 \mathrm{~Pa}$ and the residual water saturation $\left(S_{w}=0.07\right)$ is established at $P_{C} \approx-6,000 \mathrm{~Pa}$. For Toray090, water withdrawal begins at $P_{C}=-5,000 \mathrm{~Pa}$ and levels off at $S_{W}=0.04$ when $P_{C} \approx-15,000 \mathrm{~Pa}$. Upon water reinjection, a different path is followed. Some water imbibes freely into Toray090 at $P_{C}<0$, but not into SGL10BA. Subsequent water withdrawal follows the first withdrawal path exactly and all subsequent injections follow the re-injection path. These findings agree qualitatively with those of Fairweather et al. [9], but are in stark contrast with those of Nguyen et al. [10]. Water withdrawal from any $S_{w}$ reached by water injection results in hysteresis, as shown by the hysteresis loops in Figure 3. 
Hysteresis of the $P_{C}\left(S_{W}\right)$ relationship in porous media has a dual origin. Firstly, the contact angles prevailing during water injection $\left(\theta_{A}\right)$ and withdrawal $\left(\theta_{R}\right)$ are different $\left(\theta_{R}<\theta<\theta_{A}\right)$, a fact known as contact angle hysteresis [6]. Secondly, capillary equilibrium is established via irreversible meniscus transitions [11], which involve interfacial configurations that depend on the history of saturation change (water injection or withdrawal). By any account, $50^{\circ}<\theta<\theta_{A}$ for water on the carbon surfaces of GDL materials, so that water cannot spontaneously imbibe [12] into Toray090 (0\% PTFE). This cannot be anticipated from a straight capillary tube model, which predicts spontaneous pore filling for any $\theta<90^{\circ}$. In media of intermediate wettability, the usefulness of such a model to explain wettability effects is questionable because geometric effects dominate the solution to Eq.(1) [13]. Water withdrawal from a saturated GDL is controlled by capillary instabilities of anticlastic interfaces and the associated capillary pressures may be estimated from [14]:

$$
P_{C}=2 \sigma\left(-\frac{\cos \theta_{R}}{D_{p}}-\frac{\sin \theta_{R}}{\sqrt{D_{p}^{2}+3 D_{t}^{2}}}\right)
$$

where $D_{p}$ and $D_{t}$ are characteristic pore and throat diameters. Neglecting contact angle hysteresis and inserting $\theta_{R}=\theta=98^{\circ}$ into Eq.(4) with $D_{p}=19 \mu \mathrm{m}$ and $D_{t}=16 \mu \mathrm{m}$ for Toray090 [3] gives $P_{C}=-3,190 \mathrm{~Pa}$ for water withdrawal, compared to an experimentally measured value of $-5200 \mathrm{~Pa}$. This shows qualitatively that geometric effects during water retraction are significant and sufficient to generate negative capillary pressures, even when water is a non-wetting fluid $\left(\theta=98^{\circ}\right)$. Incorporating the effect of contact angle hysteresis would make Eq.(4) even more negative, bringing the calculated $P_{C}$ closer to experiment. 


\section{Conclusions}

A straightforward and accurate method for measuring air-water capillary pressure curves of GDL materials along any saturation path in the range $0 \leq S_{W} \leq 1$ has been developed. Results for both Toray090 and SGL10BA confirm significant capillary pressure hysteresis. Capillary equilibrium along paths of water injection and withdrawal is reached with $P_{C}>0$ and $P_{C}<0$, respectively, as expected for intermediately-wet materials with narrow pore size distribution and low pore-to-throat size aspect ratio. 


\section{References}

[1] H.A. Gasteiger, S.S. Kocha, B. Sompalli and F.T. Wagner, Appl. Catal. B 56 (2005) 9.

[2] R. Defay, I. Prigogine and A. Bellemans, Surface Tension and Adsorption, Longmans, 1966.

[3] J.T. Gostick, M.A. Ioannidis, M.W. Fowler and M.D. Pritzker, J. Power Sources 173 (2007) 277.

[4] V. Gurau, M.J. Bluemle, E.S. De Castro, Y.M. Tsou, J. Mann and T. Zawodzinski, J. Power Sources 160 (2003) 1156.

[5] A.W. Adamson, Physical Chemistry of Surfaces, John Wiley \& Sons, New York, 1982.

[6] F.A.L. Dullien, Porous Media: Fluid Transport and Pore Structure, Academic Press, New York, 1992.

[7] J.T. Gostick, M.W. Fowler, M.A. Ioannidis, M.D. Pritzker, Y.M. Volfkovich and A. Sakars, J. Power Sources 156 (2006) 375.

[8] T. Koido, T. Furusawa and K. Moriyama, J. Power Sources 175 (2008) 127.

[9] J.D. Fairweather, P. Cheung, J. St Pierre and D.T. Schwartz, Electrochem. Commun. 9 (2007) 2340.

[10] T.V. Nguyen, G. Lin, H. Ohn and X. Wang, Electrochem. Solid-State Lett. 11 (2008) B127.

[11] N.R. Morrow, Ind. Eng. Chem. 62 (1970) 32.

[12] N. Shirtcliffe, G. McHale, M. Newton, F. Pyatt and S. Doerr, Appl. Phys. Lett. 89 (2006) 094101.

[13] W. Anderson, J. Petro. Tech. 39 (1987) 1283.

[14] M.A. Ioannidis, I. Chatzis and A.C. Payatakes, J. Colloid Interface Sci. 143 (1991) 22. 


\section{Figures}

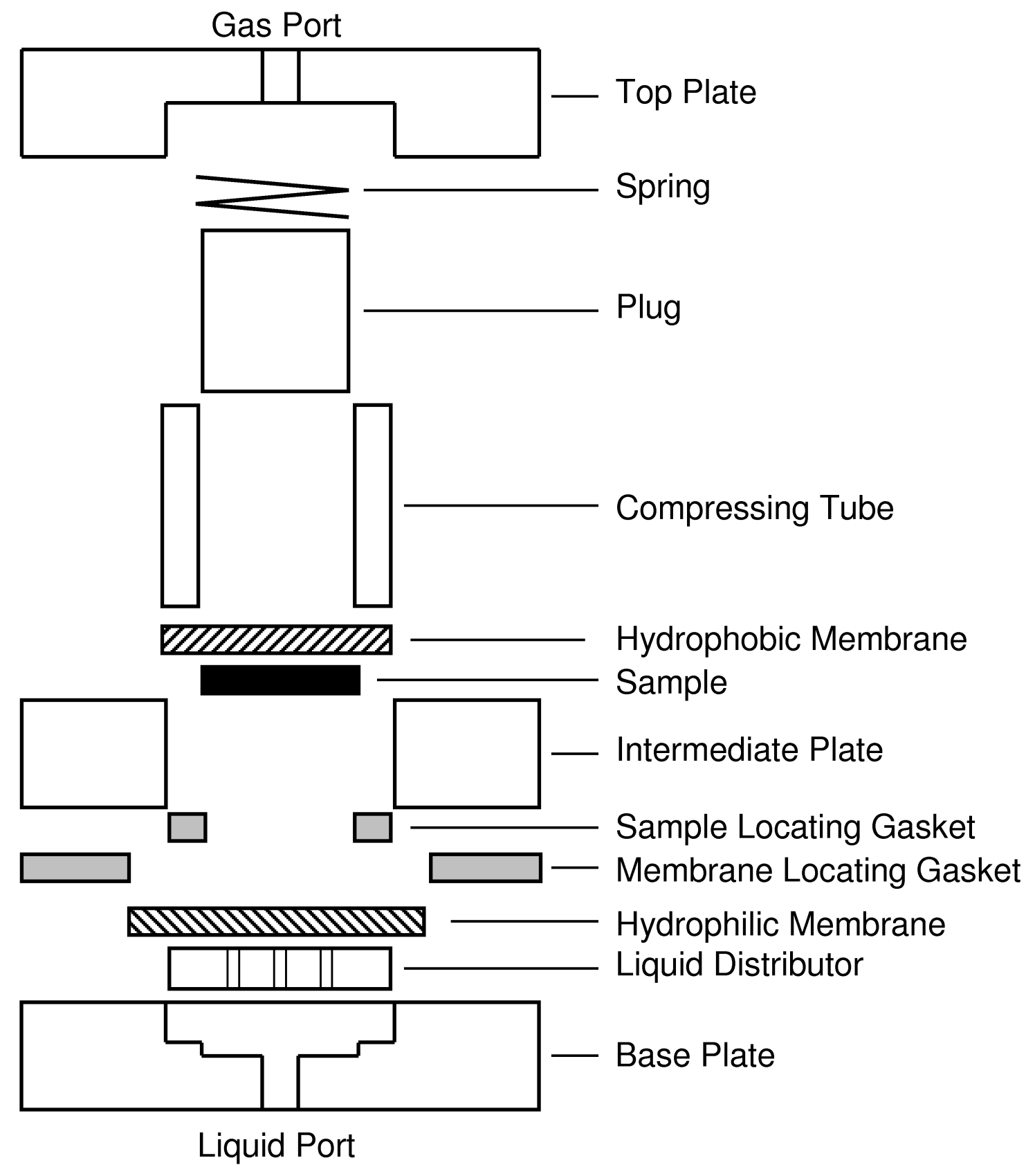

Figure 1 - Expanded view of sample holder. 


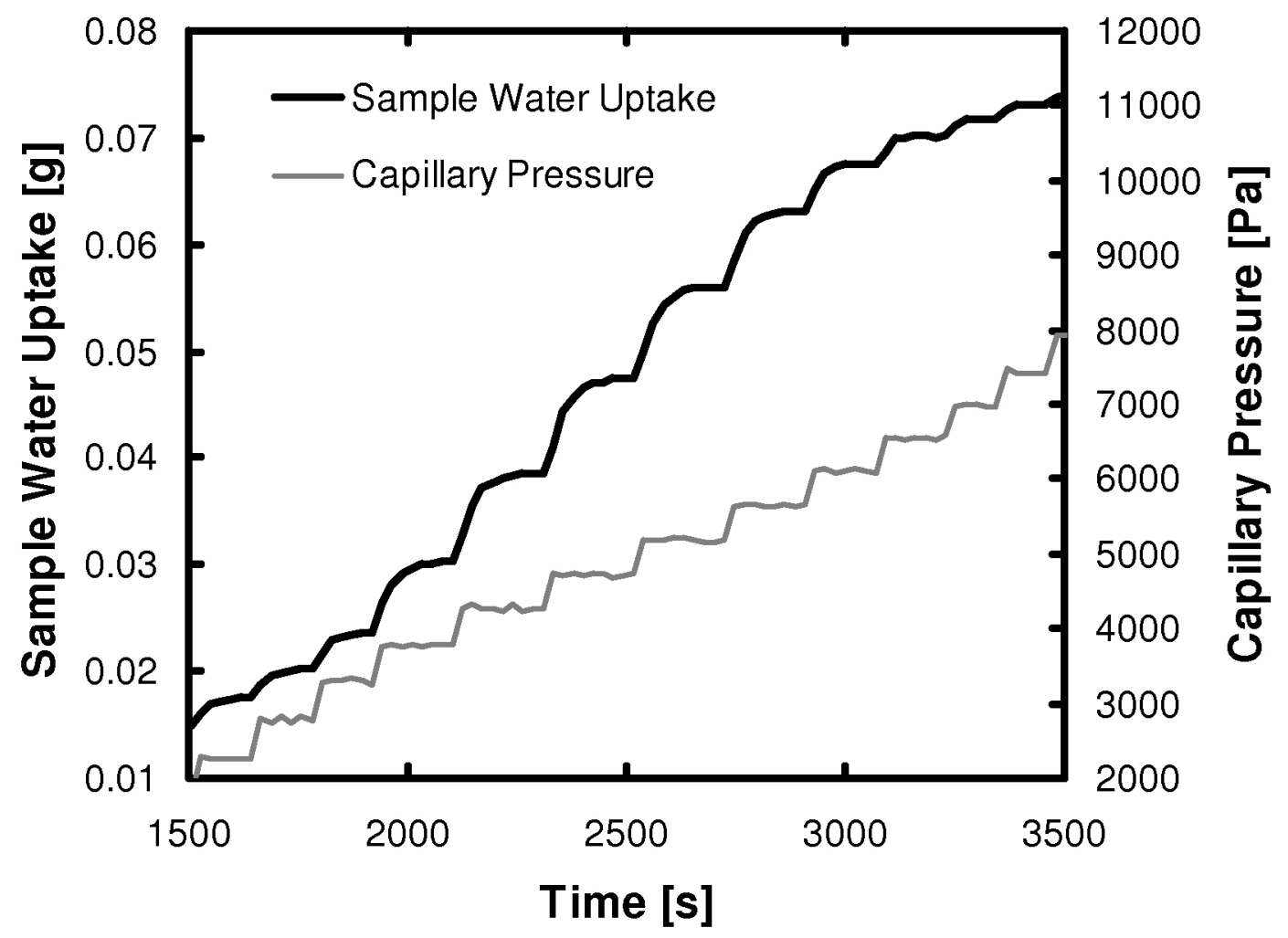

Figure 2 - Sample of raw data for SGL10BA showing transient water uptake into sample as the capillary pressure is incremented stepwise. 

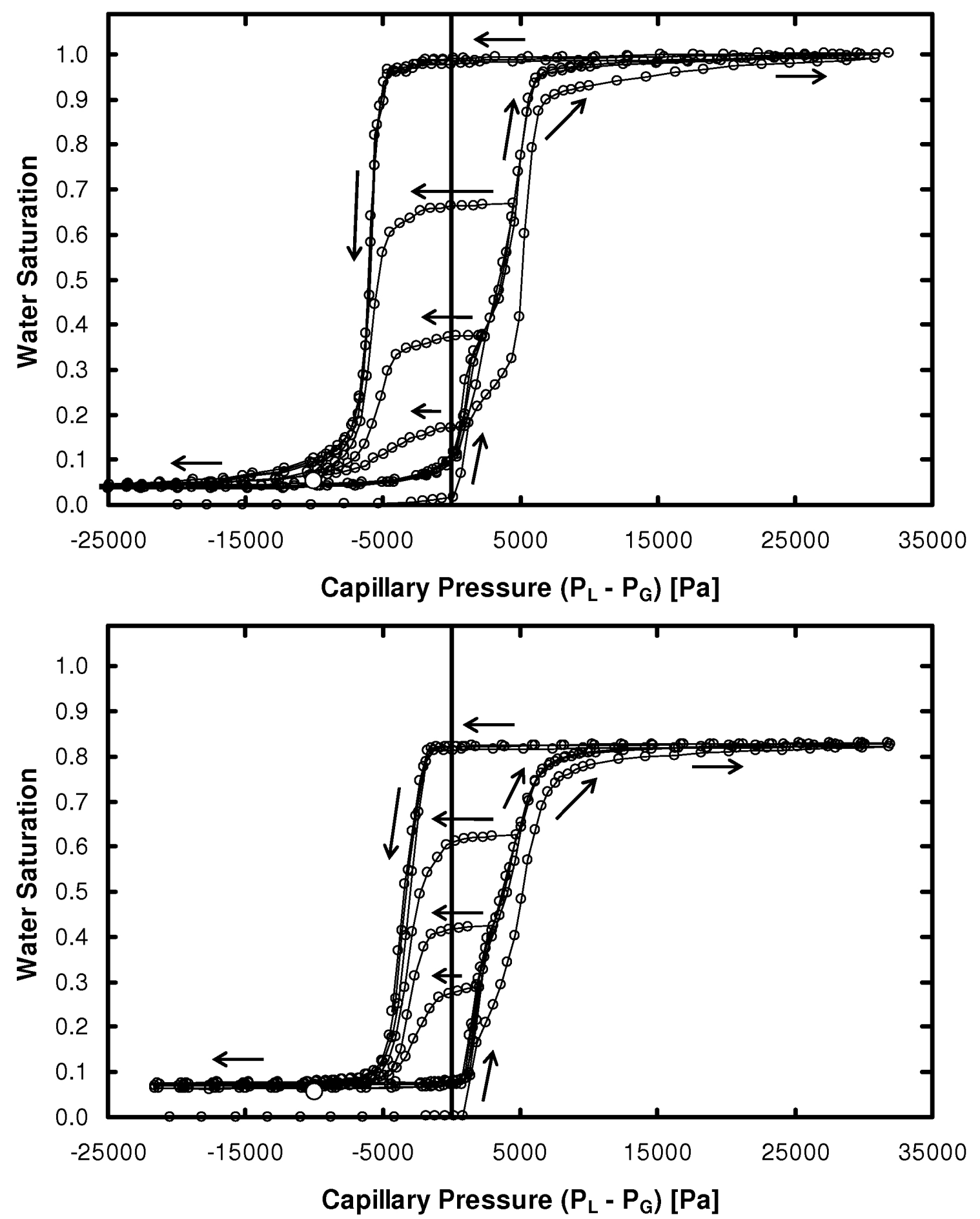

Figure 3 - Air water capillary pressure curve for Toray090 (top) and SGL10BA (bottom). The white circles at $-10,000 \mathrm{~Pa}$ are the residual saturation level determined independently by weighing the samples after the experiment. 